Internist 2022 · 63:12-17

https://doi.org/10.1007/s00108-021-01209-4

Angenommen: 27. Oktober 2021

Online publiziert: 3. Dezember 2021

๑) Der/die Autor(en) 2021

Redaktion

Martin Reincke, München

\title{
Neue Aspekte der Glukokortikoidsubstitution bei Nebennierenrindeninsuffizienz
}

\author{
Tina Kienitz ${ }^{1} \cdot$ Gesine Meyer ${ }^{2}$ \\ 'Endokrinologie in Charlottenburg, Berlin, Deutschland \\ ${ }^{2}$ Medizinische Klinik 1 - Schwerpunkt Endokrinologie, Diabetes und Stoffwechsel, Universitätsklinikum \\ Frankfurt, Frankfurt, Deutschland
}

\section{Zusammenfassung}

\section{In diesem Beitrag}

- Therapie

- Die richtige Glukokortikoiddosis

- Mineralokortikoide

- Risiko einer adrenalen Krise

- Vermeidung von Nebennierenkrisen

- Behandlung der adrenalen Krise

- Nebennierenrindeninsuffizienz und COVID-19

\section{Zusatzmaterial online}

Die Online-Version dieses Beitrags (https://doi.org/10.1007/s00108-02101209-4) enthält den vollständigen Notfallausweis für Patienten mit Nebennierenrindeninsuffizienz.

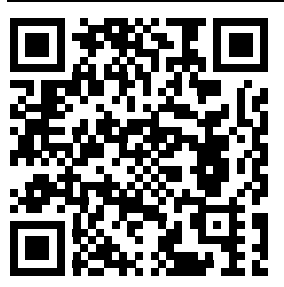

QR-Code scannen \& Beitrag online lesen

Hintergrund: Eine adäquate Anpassung der Glukokortikoidsubstitution an unterschiedliche Situationen ist essenziell für Leistungsfähigkeit und Lebensqualität von Patienten mit Nebennierenrindeninsuffizienz (NNRI). Sie dient darüber hinaus der Vermeidung lebensbedrohlicher adrenaler Krisen.

Ziel der Arbeit: Verbesserung der Versorgung von Patienten mit Nebennierenrindeninsuffizienz.

Material und Methoden: Selektive Literaturrecherche unter besonderer Berücksichtigung neuerer Studien.

Ergebnisse: Eine optimale Glukokortikoidsubstitution hat das Ziel, die physiologischen Kortisolschwankungen möglichst genau nachzuahmen. Hier haben in den letzten Jahren Präparate mit veränderter Pharmakokinetik das Therapiespektrum erweitert. Im Vordergrund stehen eine adäquate Anpassung der Substitution in Stresssituationen sowie die Vermeidung und adäquate Behandlung adrenaler Krisen, die mit einer Inzidenz von 4,8 bis 8,3 Krisen pro 100 Patientenjahre auftreten und mit einer Mortalität von etwa 0,5 pro 100 Patientenjahre weiterhin eine tödliche Bedrohung darstellen. Schlussfolgerung: Zur Verhinderung lebensbedrohlicher Nebennierenkrisen ist eine Schulung von Patienten, Angehörigen und insbesondere auch medizinischem Personal notwendig.

\section{Schlüsselwörter}

Hydrocortison · Adrenale Krise · Notfallausweis · COVID-19 · Mineralokortikoide

Grundlage der Therapie einer Nebennierenrindeninsuffizienz (NNRI) ist die Hormonersatztherapie mit Glukokortikoiden. Diese sollte die physiologischen Kortisolschwankungen im Tagesverlauf so gut wie möglich nachahmen. Erschwert wird die bedarfsgerechte Substitutionstherapie durch den erheblich gesteigerten Kortisolbedarf in Stresssituationen. Erfolgt die in solchen Situationen notwendige Anpassung nicht oder ist eine ausreichende Resorption oraler Glukokortikoide beispielsweise aufgrund eines gastrointestinalen Infekts nicht gewährleistet, besteht die Gefahr einer lebensbedrohlichen adrenalen Krise.
Die klinischen Zeichen der NNRI sind unspezifisch, was die Diagnosestellung erschwert. Von Bedeutung ist, die verschiedenen Formen der NNRI zu unterscheiden (-Tab. 1). Die häufigste Form ist die iatrogene (tertiäre) NNRI. Bereits nach wenigen Tagen einer Therapie mit 20-30 mg Prednisolonäquivalent kann die Hypothalamus-Hypophysen-Nebennieren-Achse nachhaltig gestört werden. Insbesondere hochpotente Depotpräparate und eine unphysiologische abendliche Gabe bergen das Risiko einer längerfristigen Suppression des Regelkreises [8]. 
Tab. 1 Übersicht über die verschiedenen Formen der Nebennierenrindeninsuffizienz

\begin{tabular}{|c|c|c|}
\hline Primäre NNRI & Sekundäre NNRI & Tertiäre NNRI \\
\hline $\begin{array}{l}\text { Irreversible Schädigung der } \\
\text { Nebennierenrinde }\end{array}$ & $\begin{array}{l}\text { ACTH-Mangel und konsekutiv } \\
\text { Atrophie der Nebennierenrinde }\end{array}$ & $\begin{array}{l}\text { Suppression der Hypo- } \\
\text { thalamus-Hypophysen- } \\
\text { Nebennieren-Achse }\end{array}$ \\
\hline \multicolumn{3}{|l|}{ Epidemiologie } \\
\hline $\begin{array}{l}\text { Prävalenz: 93-144/1 Mio. [2, 5, } \\
\text { 22] }\end{array}$ & $\begin{array}{l}\text { Prävalenz: 150-280/1 Mio. [22, } \\
\text { 26] }\end{array}$ & \multirow[t]{2}{*}{$\begin{array}{l}\text { Prävalenz: ca. 0,5-2,0\% } \\
\text { der Bevölkerung }[8,16]\end{array}$} \\
\hline Inzidenz: 5/1 Mio./Jahr $[2,5]$ & Inzidenz: 20/1 Mio./Jahr [26] & \\
\hline \multicolumn{3}{|l|}{ Mögliche Ursachen } \\
\hline Autoimmunadrenalitis & Tumoren der Hypophysenregion & $\begin{array}{l}\text { latrogen (länger anhalten- } \\
\text { de Glukokortikoidtherapie) }\end{array}$ \\
\hline Beidseitige Adrenalektomie & $\begin{array}{l}\text { Operationen in der Hypophy- } \\
\text { senregion }\end{array}$ & \multirow[t]{8}{*}{$\begin{array}{l}\text { Zustand nach endogenem } \\
\text { Cushing-Syndrom }\end{array}$} \\
\hline Adrenogenitales Syndrom & $\begin{array}{l}\text { Bestrahlung der Hypophysenre- } \\
\text { gion }\end{array}$ & \\
\hline $\begin{array}{l}\text { Infektionen (Tuberkulose, CMV, } \\
\text { HIV, Mykosen) }\end{array}$ & $\begin{array}{l}\text { Hypophyseninfarkt/Sheehan- } \\
\text { Syndrom }\end{array}$ & \\
\hline $\begin{array}{l}\text { Beidseitige Nebenniereneinblu- } \\
\text { tungen (Meningokokkensepsis) }\end{array}$ & Autoimmunhypophysitis & \\
\hline $\begin{array}{l}\text { Beidseitige Nebennierenmetas- } \\
\text { tasen }\end{array}$ & $\begin{array}{l}\text { Granulomatöse Erkrankungen } \\
\text { (Sarkoidose, Histiozytose X) }\end{array}$ & \\
\hline Adrenoleukodystrophie & $\begin{array}{l}\text { Infektionen (tuberkulöse Me- } \\
\text { ningitis) }\end{array}$ & \\
\hline \multirow[t]{2}{*}{ Genetische Ursachen } & Schädel-Hirn-Trauma & \\
\hline & Genetische Ursachen & \\
\hline
\end{tabular}

\section{Therapie}

Grundlage der Therapie einer NNRI ist die Hormonersatztherapie mit Glukokortikoiden. Das am häufigsten eingesetzte Glukokortikoid in Deutschland ist Hydrocortison (HC). Übliche Tagessubstitutionsdosen in Deutschland liegen bei $10-30 \mathrm{mg}$ [27]. Die Einnahme sollte in 2-3 Einzeldosen erfolgen mit etwa zwei Drittel der Dosis beim Aufwachen am frühen Morgen und einem Drittel 6-8h später (beispielsweise HC 1510-0-0 mg oder 10-10-5-0 mg). Patienten mit sekundärer NNRI benötigen in der Regel niedrigere Substitutionsdosen.

Eine Substitution mit Prednisolon ist möglich, jedoch müssen die längere Halbwertszeit und stärkere Glukokortikoidpotenz im Vergleich zu HC (etwa 1:5) beachtet werden. Üblich ist eine 1-mal tägliche Einnahme von 3 bis $5 \mathrm{mg}$ Prednisolon am Morgen [7]. Prednisolon hat gegenüber HC ein ähnliches kardiovaskuläres Risikoprofil [30], jedoch negative Einflüsse auf Low-density-Lipoprotein-CholesterinWerte [28] und Knochendichte [11].
Seit 2012 ist HC mit 2-stufiger Wirkstofffreisetzung (Plenadren ${ }^{\circledR}$, verfügbar als 5- und $20 \mathrm{mg}$-Tabletten) zur Therapie der NNRI bei Erwachsenen zugelassen. Die veränderte Pharmakokinetik beruht auf einer schnellen Freisetzung von $\mathrm{HC}$ aus der Hülle des Medikaments und einer verzögerten Freisetzung aus dem Kern. In der randomisierten, kontrollierten $\mathrm{Zu}$ lassungsstudie konnte eine Verbesserung von Lebensqualität, Blutdruck und Glukosetoleranz erzielt werden. In weiteren, offenen klinischen Studien konnte die Verbesserung von Lebensqualität und glykometabolischem Profil bei Patienten mit primärer NNRI bestätigt werden [4, 24], während Daten zum metabolischen Profil von Patienten mit sekundärer NNRI divergieren [24, 32]. Die Tagestherapiekosten liegen deutlich über denen einer konventionellen Therapie mit HC.

Im Sommer 2021 wurde HC mit verzögerter Wirkstofffreisetzung (Efmody ${ }^{\circledR}$, verfügbar als 5- und $10 \mathrm{mg}$-Tabletten) für $\mathrm{Pa}$ tienten ab 12 Jahren mit adrenogenitalem Syndrom zugelassen. Eine spezielle Formulierung von HC führt bei dem Me- dikament zu einer verzögerten Absorption. Die Einnahme erfolgt um 22 Uhr, die Wirkung entfaltet sich in den frühen Morgenstunden. Hierdurch lässt sich die Androgenbildung effektiver supprimieren. Zwar waren in der randomisierten PhaseIII-Zulassungsstudie über 6 Monate keine Unterschiede hinsichtlich der Kontrolle der 17-Hydroxy-Progesteron(17-OHP)-Serumspiegel über $24 \mathrm{~h}$ zu sehen, die morgendlichen 17-OHP-Serumspiegel ließen sich jedoch mit dem neuen Präparat besser supprimieren. Auch war in der 1-armigen Extensionsstudie eine Reduktion der HCTagesdosis möglich [21].

\section{॥) Übliche Tagessubstitutionsdosen liegen bei 10-30 mg Hydrocortison (HC)}

Durch eine Pumpentherapie mit HC lässt sich die Glukokortikoidsubstitution dem physiologischen Profil am besten annähern. Mittels kontinuierlicher subkutaner HC-Infusion lässt sich eine verbesserte Lebensqualität erzielen [25]. Andere anthropometrische Parameter oder klinische Parameter zeigten jedoch gegenüber der 3-mal täglichen oralen Einnahme keine Unterschiede [6]. Patienten mit nur geringer Einschränkung ihrer Lebensqualität scheinen nicht zu profitieren [12]. Insbesondere für Patienten mit primärer NNRI und stark fluktuierenden Energieniveaus während des Tages unter konventioneller Therapie könnte eine Pumpentherapie jedoch eine Option darstellen. In Deutschland ist der Einsatz nur im Rahmen klinischer Studien und individueller Einzelfallentscheidungen möglich.

\section{Die richtige Glukokortikoiddosis}

Die endogene Kortisolsekretion folgt einer zirkadianen Rhythmik mit dem höchsten Spiegel in den frühen Morgenstunden (gegen $8 \mathrm{Uhr}$ ) und einem etwas kleineren Peak am frühen Nachmittag (gegen $14 \mathrm{Uhr}$ ). Am niedrigsten ist die Kortisolsekretion um Mitternacht. Stress, Infektionen oder Verletzungen erhöhen die endogene Kortisolproduktion um ein Vielfaches. Während einerseits eine chronische Übersubstitution vermieden werden muss, muss andererseits ein akut vermehrter Kortisolbedarf zwingend ausgeglichen werden. Die Güte 


\begin{tabular}{|l|l|}
\hline Tab. 2 Zeichen und Symptome der Über-bzw. Untersubstitution mit Glukokortikoiden \\
\hline Übersubstitution & Untersubstitution \\
\hline Gewichtszunahme & Gewichtsabnahme \\
\hline $\begin{array}{l}\text { Cushingoide Zeichen: Facies lunata, Hautatrophie, } \\
\text { Muskelschwäche, nuchales und supraklavikuläres } \\
\text { Fettpolster }\end{array}$ & Hypotonie und Schwindel \\
\cline { 2 - 2 } & Müdigkeit und Erschöpfung \\
\hline Glukoseintoleranz/Diabetes mellitus & Übelkeit und Erbrechen \\
\hline Osteopenie/Osteoporose & Hyponatriämie und/oder Hypoglykämie \\
\hline Hypertonie & $\begin{array}{l}\text { Hyperpigmentierung (bei primärer Ne- } \\
\text { bennierenrindeninsuffizienz) }\end{array}$ \\
\hline
\end{tabular}

Tab. 4 Dosisanpassung der Glukokortikoidsubstitution in Situationen, in denen eine Kortisolunterversorgung droht, gemäß den Empfehlungen im Notfallausweis des Netzwerks für Hypophysen- und Nebennierenerkrankungen e. V. in Zusammenarbeit mit der Deutschen Gesellschaft für Endokrinologie

\begin{tabular}{|c|c|}
\hline Situation & Empfehlung \\
\hline Leichte Verletzungen & \multirow{2}{*}{$\begin{array}{l}\text { Gegebenenfalls zusätzliche Einnah- } \\
\text { me von } 5 \text { bis } 10 \mathrm{mg} \mathrm{HC}\end{array}$} \\
\hline Anstrengende Aktivitäten über das Gewohnte hinaus & \\
\hline $\begin{array}{l}\text { Infekt mit leichtem bis mittlerem Krankheitsgefühl ohne } \\
\text { Fieber oder } \\
\text { deutliche Belastungssituation (starke körperliche Belas- } \\
\text { tung, starker Schmerz, Zahneingriffe, kleinere ambulan- } \\
\text { te Eingriffe, erhebliche psychische Belastung, z. B. bei } \\
\text { Trauerfall, Prüfung, Hochzeit) }\end{array}$ & $\begin{array}{l}\text { Tagesdosis verdoppeln, gegebenen- } \\
\text { falls zusätzlich abends } 5-10 \mathrm{mg} \mathrm{HC}\end{array}$ \\
\hline \multirow[t]{2}{*}{$\begin{array}{l}\text { Akute Erkrankungen und/oder Fieber mit deutlichem } \\
\text { Krankheitsgefühl }\end{array}$} & $\begin{array}{l}\text { Tagesdosis verdreifachen oder } \\
30-20-10 \mathrm{mg} \mathrm{HC} \text { (bei Tagesdosis } \\
\leq 20 \mathrm{mg} \text { ) }\end{array}$ \\
\hline & Dringend ärztliche Hilfe holen \\
\hline \multirow[t]{2}{*}{$\begin{array}{l}\text { Anhaltendes Erbrechen und/oder Durchfall oder hohes } \\
\text { Fieber }\left(>39^{\circ} \mathrm{C}\right) \text { mit schwerem Krankheitsgefühl }\end{array}$} & $\begin{array}{l}100 \mathrm{mg} \mathrm{HC} \text { (oder anderes Glukokorti- } \\
\text { koid) parenteral }\end{array}$ \\
\hline & Sofort ärztliche Hilfe holen! \\
\hline \multirow[t]{2}{*}{ Operationen (stationär, Vollnarkose) } & $\begin{array}{l}\text { Operationstag: } 100 \mathrm{mg} \mathrm{HC} \text { i.v. als } \\
\text { Bolus vor Narkoseeinleitung, gefolgt } \\
\text { von } 100 \text { bis } 200 \mathrm{mg} / 24 \mathrm{~h} \text { i.v. }\end{array}$ \\
\hline & $\begin{array}{l}\text { Postoperativ: } 100 \mathrm{mg} / 24 \mathrm{~h} \text { i.v., bis } \\
\text { Patient essen und trinken darf, dann } \\
\text { Umstellung auf doppelte bis 3-fa- } \\
\text { che Tagesdosis für } 24-48 \mathrm{~h} \text {, weitere } \\
\text { Reduktion nach Verlauf und Klinik }\end{array}$ \\
\hline C Hydrocortis & \\
\hline
\end{tabular}

der Glukokortikoidsubstitution lässt sich nicht anhand laborchemischer Parameter überwachen. Die Einstellung obliegt der klinischen Einschätzung sowie dem Suchen nach Zeichen und Symptomen der Über- bzw. Untersubstitution (- Tab. 2).

\section{Mineralokortikoide}

Bei der primären NNRI muss zusätzlich eine Mineralokortikoidsubstitution mit 0,05-0,15 mg Fludrocortison 1-mal täglich erfolgen. Anders als bei der Glukokortikoidsubstitution gibt es klinische Kontrollund laborchemische Verlaufsparameter (Anstreben einer Normotonie, Serum- natrium und -kalium im Normbereich, Renin). Bei erhöhtem Blutdruck muss eine Reduktion, bei sehr hohen Temperaturen oder in der Schwangerschaft eine Steigerung der Fludrocortisondosis erwogen werden.

\section{Risiko einer adrenalen Krise}

Eine akute Unterversorgung mit Kortisol ist ein potenziell lebensbedrohliches Krankheitsbild. Sie kann zu

- Hypotonie bis hin zum Schock,

- Elektrolytentgleisungen mit Hyponatriämie und Hyperkaliämie,

- Hypoglykämien,
Tab. 3 Mögliche Auslöser einer adrenalen Krise. (Nach [14])

\begin{tabular}{|l|l|}
\hline Auslöser & $\begin{array}{l}\text { Häufig- } \\
\text { keit (\%) }\end{array}$ \\
\hline Gastrointestinale Infekte & $22-33$ \\
\hline Fieberhafte Erkrankungen & $17-24$ \\
\hline $\begin{array}{l}\text { Unzureichende Anpassung der } \\
\text { Substitution bei einem operati- } \\
\text { ven oder invasiven Eingriff }\end{array}$ & $7-16$ \\
\hline Intensive körperliche Belastung & $7-8$ \\
\hline Erhebliche psychische Belastung & $4-6$ \\
\hline
\end{tabular}

- prärenalen Nierenfunktionseinschränkungen bis hin zum Nierenversagen sowie

- Bewusstseinseinschränkungen

führen.

॥) Trotz der guten Behandlungsmöglichkeit sind Nebennierenkrisen weiterhin eine tödliche Bedrohung

Definiert wird eine adrenale Krise als akute Verschlechterung des Gesundheitszustands mit mindestens 2 der folgenden Symptome:

- Ausgeprägte Schwäche

- Übelkeit und/oder Erbrechen

- Hypotonie (systolischer Blutdruck $<100 \mathrm{~mm} \mathrm{Hg}$ )

- Dokumentierte Hyponatriämie und/ oder Hyperkaliämie und/oder Hypoglykämie

Es besteht die Notwendigkeit zur parenteralen Glukokortikoidgabe [1].

Die Inzidenz ist mit 4,8-8,3 Krisen pro 100 Patientenjahre höher als früher vermutet $[15,22,29]$. Das Risiko ist bei primärer NNRI mehr als doppelt so hoch wie bei sekundärer NNRI. Trotz der prinzipiell guten Behandlungsmöglichkeitstellen Krisen weiterhin eine tödliche Bedrohung dar mit einer Mortalität von etwa 0,5 pro 100 Patientenjahre [15]. Auslöser einer adrenalen Krise sind in - Tab. 3 zusammengefasst.

\section{Vermeidung von Nebennieren- krisen}

Um das Auftreten einer adrenalen Krise zu vermeiden, müssen Patienten ihre Substitutionsdosis eigenständig rasch und adäquat an den aktuellen Bedarf anpassen. Seit 2014 bietet die Deutsche Gesellschaft 


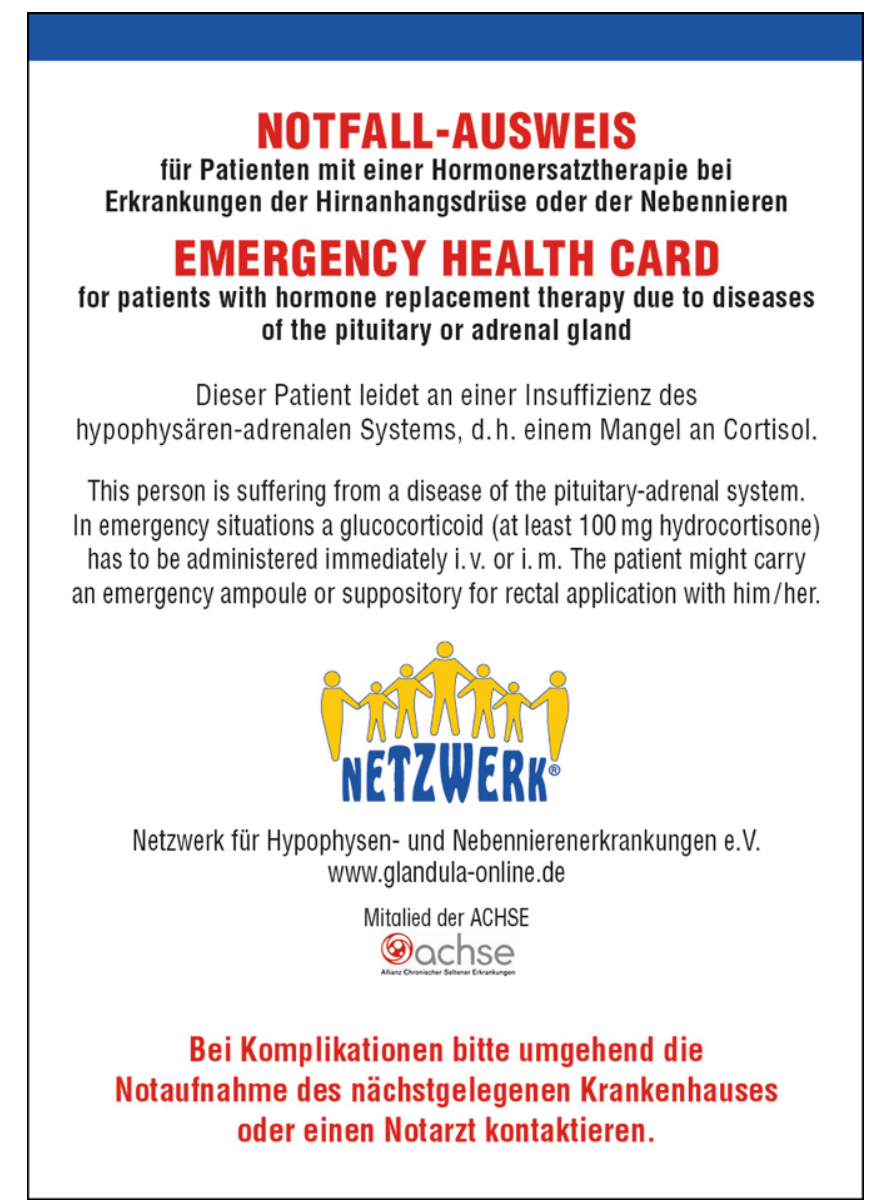

Abb. $1 \varangle$ Notfallausweis für Patienten mit Nebennierenrindeninsuffizienz [31]. Der vollständige Ausweis ist im Online-Zusatzmaterial zu finden. (Aus [31]; mit freundl. Genehmigung $\odot$ Netzwerk für Hypophysenund Nebennierenerkrankungen e. V. alle Rechte vorbehalten) für Endokrinologie ein strukturiertes und einheitliches Curriculum zur Schulung von Patienten mit NNRI und ihrer Angehörigen an. Mittlerweile findet diese Schulung regelmäßig in vielen endokrinologischen Zentren statt und zeigt positive Auswirkungen $[9,10]$. Alle Betroffenen müssen mit einem Notfallausweis (• Abb. 1) ausgestattet werden, der die wichtigsten Informationen zum Vorgehen bei Erkrankung, Unfall und Operation sowie im Falle einer adrenalen Krise beinhaltet (• Tab. 4).

Bei invasiven Eingriffen sowie während einer Entbindung ist eine entsprechend hoch dosierte parenterale Substitution durch den Behandler zu gewährleisten [1, 7]. Für kleinere ambulante Eingriffe in Lokalanästhesie, wie zahnärztliche Eingriffe oder Exzisionen von Hautveränderungen, genügt in aller Regel eine Verdopplung der oralen Dosis (- Tab. 4).

Auch die für eine Koloskopie notwendigen Abführmaßnahmen können prinzipiell zur Entstehung einer adrenalen Krise führen. Es sollten daher isoosmotische Abführpräparate mit einem möglichst gerin- gen Risiko von Flüssigkeits- und Elektrolytverschiebungen zum Einsatz kommen. Insbesondere bei älteren und multipel vorerkrankten Patienten sind eine Durchführung der abführenden Maßnahmen unter stationärer Überwachung und eine parenterale Gabe von HC (beispielsweise $50 \mathrm{mg}$ i.v. alle $8 \mathrm{~h}$ ab Beginn der Abführmaßnahmen) zu empfehlen.

\section{Behandlung der adrenalen Krise}

Der Übergang von einer drohenden zu einer manifesten adrenalen Krise verläuft fließend. Bereits eine drohende Krise ist ein medizinischer Notfall, der rasches Handeln erfordert. Die wichtigste therapeutische Maßnahme ist die intravenöse Zufuhr von Glukokortikoiden und Flüssigkeit unter engmaschigem Monitoring.

Rasches Handeln rettet bei einer drohenden oder bereits manifesten adrenalen Krise Leben und gefährdet die Patienten auch dann nicht, wenn die Situation anfangs überschätzt wurde und keine adrenale Krise vorliegt.
Akuttherapie (nach [7]):

- $100 \mathrm{mg} \mathrm{HC}$ i.v. als Bolus

- Gefolgt von $200 \mathrm{mg} / \mathrm{Tag}$ als $24 \mathrm{~h}$ Dauerinfusion oder regelmäßige Gabe eines Bolus von 50 bis $100 \mathrm{mg}$ alle $6 \mathrm{~h}$

- Wenn HC nicht verfügbar ist: 25 mg Prednisolon i.v. als Bolus, gefolgt von regelmäßigen Boli. Cave: Bei Verwendung von Glukokortikoiden ohne ausreichende Mineralokortikoidwirkung bei primärer NNRI zusätzliche Applikation von 0,05 bis $0,15 \mathrm{mg}$ Fludrocortison p.o. pro Tag

- Infusion von balancierter Elektrolytlösung oder $5 \%$ iger Glukoselösung in isotonischer Kochsalzlösung mit einer anfänglichen Infusionsrate von $1 \mathrm{l} / \mathrm{h}$, Anpassung der Infusionsrate nach Stabilisierung

- Intensivmedizinische Überwachung mit engmaschigem hämodynamischem Monitoring

- Engmaschige Elektrolytkontrollen (insbesondere Natrium, Kalium, Glukose) und gegebenenfalls Ausgleich

Das Erleben einer adrenalen Krise und auch bereits die Angst vor dieser Komplikation belasten die Betroffenen erheblich [23]. Verstärkt wird dies durch eine verzögerte Erkennung [17] und Therapie [13] durch medizinisches Fachpersonal. Leider berichten Patienten nicht selten von Situationen, in denen auch nach Vorlage des Notfallausweises eine adäquate Hilfe erst nach deutlicher Zeitverzögerung, teilweise auch gar nicht erfolgte.

\section{Nebennierenrindeninsuffizienz und COVID-19}

Die Bedrohung durch eine potenziell lebensbedrohlich verlaufende Infektion mit dem "severe acute respiratory syndrome coronavirus 2" (SARS-CoV-2) führt seit Beginn der Pandemie bei Patienten mit NNRI zu einer teilweise erheblichen zusätzlichen Belastung [20]. Es ist wenig darüber bekannt, ob das Risiko einer Ansteckung und/ oder des schweren Verlaufs einer "coronavirus disease 2019" (COVID-19) bei vorliegender NNRI signifikant erhöht ist. Bei Patienten mit primärer NNRI konnte eine verminderte zytotoxische Aktivität natürlicher Killerzellen gezeigt werden, die insbesondere für die Erkennung und Eliminie- 
rung virusinfizierter Zellen benötigt werden. Als Ursache dieser Beeinträchtigung des angeborenen Immunsystems wird am ehesten die trotz aller Bemühungen letztlich unphysiologisch bleibende Substitution mit Glukokortikoiden vermutet, die zu einer veränderten peripheren CLOCKGenregulation in den Immunzellen führt [3]. Vor diesem Hintergrund erscheinen ein höheres Risiko viraler Infektionen und auch ein schwererer Verlauf solcher Infektionen bei NNRI durchaus möglich - auch bei Gabe einer physiologischen Substitutionsdosis. COVID-19 kann darüber hinaus eine adrenale Krise auslösen, insbesondere bei deutlichen Symptomen, hohem Fieber und/oder gastrointestinalen Symptomen. Wie bei anderen Infektionen ist daher eine rasche Dosisanpassung und gegebenenfalls parenterale Substitution auch bei COVID-19 von höchster Wichtigkeit.

\section{॥) Patienten mit Nebennieren- rindeninsuffizienz sollten gegen COVID-19 geimpft werden}

Patienten mit Nebennierenrindeninsuffizienz sollten gegen COVID-19 geimpft werden [19]. Eine Beeinträchtigung der Impfwirkung ist unter den üblichen Substitutionsdosen nicht zu erwarten. Valide Daten hierzu liegen bislang nicht vor, bei gängigen Impfungen werden jedoch bis zu einer Tagesdosis von 10 bis $20 \mathrm{mg}$ Prednisolon keine relevanten Beeinträchtigungen des Impferfolgs beobachtet. Die Impfung sollte unter der üblichen Substitutionsdosis erfolgen. Im Falle des Auftretens einer Impfreaktion, insbesondere bei Fieber, muss die Dosis analog zu den Empfehlungen für Infekte passager erhöht werden [18].

\section{Fazit für die Praxis}

- Bei unspezifischen Symptomen wie Müdigkeit, Erschöpfung, Gewichtsverlust, Hypotonie oder Hyponatriämie gilt es, eine Nebennierenrindeninsuffizienz (NNRI) differenzialdiagnostisch auszuschließen.

- Am häufigsten ist die iatrogene NNRI nach Pharmakotherapie mit Glukokortikoiden.

- Eine optimale Glukokortikoidsubstitution hat das Ziel, die physiologischen Kortisolschwankungen möglichst genau nachzuahmen. In diesem Zusammenhang haben in den letzten Jahren Präparate mit veränderter Pharmakokinetik das Therapiespektrum erweitert.
- Nicht wenige Patienten berichten von Situationen, in denen auch nach Vorlage des Notfallausweises eine adäquate Hilfe erst nach deutlicher Zeitverzögerung, teilweise auch gar nicht erfolgte.

- Zur Verhinderung lebensbedrohlicher Nebennierenkrisen ist eine Schulung von $\mathrm{Pa}$ tienten, Angehörigen und auch medizinischem Personal notwendig.

- Patienten mit NNRI sollten alle empfohlenen Impfungen erhalten - auch gegen COVID-19.

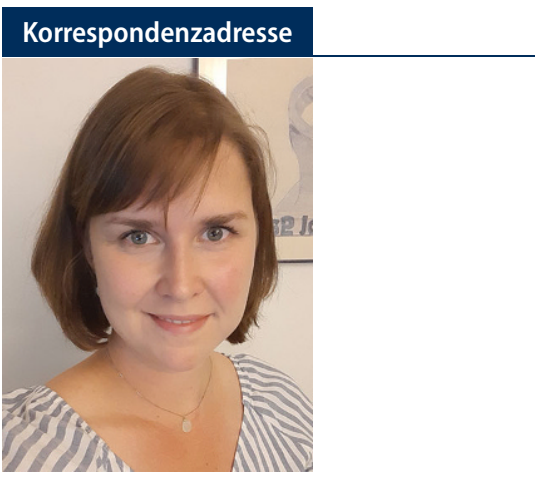

Dr. med. Tina Kienitz

Endokrinologie in Charlottenburg Stuttgarter Platz 1, 10627 Berlin, Deutschland tina.kienitz@gmail.com

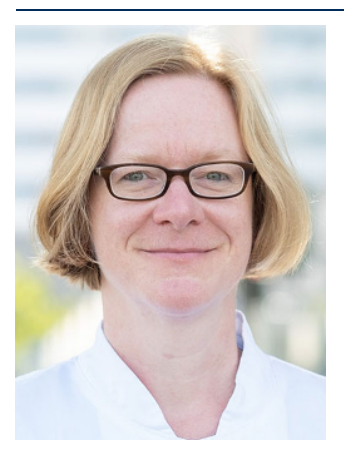

PD Dr. med. Gesine Meyer

Medizinische Klinik 1 - Schwerpunkt Endokrinologie, Diabetes und Stoffwechsel, Universitätsklinikum Frankfurt Theodor-Stern-Kai 7, 60590 Frankfurt, Deutschland

gesine.meyer@kgu.de

\section{Einhaltung ethischer Richtlinien}

Interessenkonflikt. T. Kienitz und G. Meyer erhielten Honorare für eine Beratertätigkeit von Shire.

Für diesen Beitrag wurden von den Autorinnen keine Studien an Menschen oder Tieren durchgeführt. Für die aufgeführten Studien gelten die jeweils dort angegebenen ethischen Richtlinien.

Open Access. Dieser Artikel wird unter der Creative Commons Namensnennung 4.0 International Lizenz veröffentlicht, welche die Nutzung, Vervielfältigung, Bearbeitung, Verbreitung und Wiedergabe in jeglichem Medium und Format erlaubt, sofern Sie den/die ursprünglichen Autor(en) und die Quelle ordnungsgemäß nennen, einen Link zur Creative Commons Lizenz beifügen und angeben, ob Änderungen vorgenommen wurden.

Die in diesem Artikel enthaltenen Bilder und sonstiges Drittmaterial unterliegen ebenfalls der genannten Creative Commons Lizenz, sofern sich aus der Abbildungslegende nichts anderes ergibt. Sofern das betreffende Material nicht unter der genannten Creative Commons Lizenz steht und die betreffende Handlung nicht nach gesetzlichen Vorschriften erlaubt ist, ist für die oben aufgeführten Weiterverwendungen des $\mathrm{Ma}$ terials die Einwilligung des jeweiligen Rechteinhabers einzuholen.

Weitere Details zur Lizenz entnehmen Sie bitte der Lizenzinformation auf http://creativecommons.org/ licenses/by/4.0/deed.de.

\section{Literatur}

1. Allolio B (2015) Extensive expertise in endocrinology. Adrenal crisis. Eur JEndocrinol 172:R115-R124

2. ArltW, Allolio B (2003) Adrenal insufficiency. Lancet 361(9372):1881-1893. https://doi.org/10.1016/ S0140-6736(03)13492-7

3. Bancos I, Hazeldine J, Chortis Vet al (2017) Primary adrenal insufficiency is associated with impaired natural killer cell function: a potential link to increased mortality. Eur J Endocrinol 176:471-480

4. Bannon C, Border D, Hanson P et al (2021) Early metabolic benefits of switching hydrocortisone to modified release hydrocortisone in adult adrenal insufficiency. Front Endocrinol 12:641247

5. Betterle C, Morlin L (2010) Autoimmune addison's disease. Endocr Dev 20:161-172

6. Björnsdottir S, Øksnes M, Isaksson M et al (2015) Circadian hormone profiles and insulin sensitivity in patients with Addison's disease: a comparison of continuous subcutaneoushydrocortisoneinfusion with conventional glucocorticoid replacement therapy. Clin Endocrinol 83:28-35

7. Bornstein SR, Allolio B, Arlt Wet al (2016) Diagnosis and treatment of primary adrenal insufficiency: an endocrine society clinical practice guideline. J Clin Endocrinol Metab 101:364-389

8. Broersen LHA, Pereira AM, Jørgensen JOL, Dekkers OM (2015) Adrenal insufficiency in corticosteroids use: systematic review and metaanalysis. JClin Endocrinol Metab 100:2171-2180

9. Burger-Stritt S, Eff A, Quinkler M et al (2020) Standardised patient education in adrenal insufficiency-a prospective multi-centre evaluation. Eur JEndocrinol 183:119-127

10. Burger-Stritt S, Kardonski P, Pulzer A et al (2018) Management of adrenal emergencies in educated patients with adrenal insufficiency-A prospective study. Clin Endocrinol 89:22-29

11. Frey KR, Kienitz T, Schulz J et al (2018) Prednisolone is associated with a worse bone mineral density in primary adrenal insufficiency. Endocr Connect 7:811-818

12. Gagliardi L, Nenke MA, Thynne TRJ et al (2014) Continuous subcutaneous hydrocortisone infusion therapy in Addison's disease: a randomized, placebo-controlled clinical trial. J Clin Endocrinol Metab 99:4149-4157

13. Hahner S, Hemmelmann N, Quinkler $M$ et al (2015) Timelines in the management of adrenal 
crisis - targets, limits and reality. Clin Endocrinol 82:497-502

14. Hahner S, Loeffler M, Bleicken B et al (2010) Epidemiology of adrenal crisis in chronic adrenal insufficiency: the need for new prevention strategies. Eur J Endocrinol 162:597-602

15. Hahner S, Spinnler C, Fassnacht Met al (2015) High incidence of adrenal crisis in educated patients with chronic adrenal insufficiency: a prospective study. J Clin Endocrinol Metab 100:407-416

16. Henzen C, Suter A, Lerch E et al (2000) Suppression and recovery of adrenal response after shortterm, high-dose glucocorticoid treatment. Lancet 355:542-545

17. Kampmeyer D, Lehnert $H$, Moenig $\mathrm{H}$ et al (2016) A strong need for improving the education of physicians on glucocorticoid replacement treatment in adrenal insufficiency: An interdisciplinary and multicentre evaluation. Eur J Intern Med 33:e13-e15

18. Katznelson L, Gadelha M (2021) Glucocorticoid use in patients with adrenal insufficiency following administration of the COVID-19 vaccine: a pituitary society statement. Pituitary 24:143-145

19. Ku C, Jung K, Ahn C et al (2021) COVID-19 vaccination for endocrine patients: a position statement from the Korean endocrine society. Endocrinol Metab 36:757-765

20. LiD, Genere N, Behnken Eetal (2020) Determinants of self-reported health outcomes in adrenal insufficiency: a multi-site survey study. J Clin Endocrinol Metab 106(3):e1408-e1419. https:// doi.org/10.1210/clinem/dgaa668

21. Merke DP, Mallappa A, Arlt W et al (2021) Modified-release hydrocortisone in congenital adrenal hyperplasia. J Clin Endocrinol Metab 106:e2063-e2077

22. Meyer G, Badenhoop K, Linder R (2016) Addison's disease with polyglandular autoimmunity carries a more than 2-5-fold risk for adrenal crises: German Health insurance data 2010-2013. Clin Endocrinol 85:347-353

23. Meyer G, Koch M, Herrmann E et al (2018) Longitudinal AddiQoL scores may identify higher risk for adrenal crises in Addison's disease. Endocrine 60:355-361

24. Mongioì LM, Condorelli RA, La Vignera S, Calogero AE (2018) Dual-release hydrocortisone treatment: glycometabolic profile and healthrelated quality of life. Endocr Connect 7:211-219

25. Øksnes $M$, Björnsdottir $S$, Isaksson $M$ et al (2014) Continuous subcutaneous hydrocortisone infusion versus oral hydrocortisone replacement for treatment of addison's disease: A randomized clinical trial. J Clin Endocrinol Metab 99:1665-1674

26. Paragliola R, Corsello S (2018) Secondary adrenal insufficiency: from the physiopathology to the possible role of modified-release hydrocortisone treatment. Minerva Endocrinol 43:183-197

27. Petersenn S, Honegger J, Quinkler M (2017) National German audit of diagnosis, treatment, and teaching in secondary adrenal insufficiency. Horm Metab Res 49:580-588

28. Quinkler M, Ekman B, Marelli C et al (2017) Prednisolone is associated with a worse lipid profile than hydrocortisone in patients with adrenal insufficiency. Endocr Connect 6:1-8

29. Smans LCCJ, Van Der Valk ES, Hermus ARMM, Zelissen PMJ (2016) Incidence of adrenal crisis in patients with adrenal insufficiency. Clin Endocrinol 84:17-22

30. Smith DJF, Prabhudev H, Choudhury S, Meeran K (2017) Prednisolone has the same cardiovascular

\section{New aspects of glucocorticoid substitution in adrenal insufficiency}

Background: Appropriate glucocorticoid dose adjustment in specific situations significantly impacts quality of life and performance of patients with adrenal insufficiency. It is also pivotal for the prevention of adrenal crisis.

Objectives: Improving medical care for patients with adrenal insufficiency. Materials and methods: Selective literature research focussing on the most recent studies.

Results: Optimal glucocorticoid substitution aims at closely mimicking physiological fluctuations of cortisol levels. In recent years glucocorticoid preparations with modified pharmacokinetics have expanded the therapeutic arsenal. Adrenal crises occur with an incidence of 4.8-9.3 crises per 100 patient years. With a mortality of 0.5 per 100 patient years adrenal crisis is a life-threatening event. Therefore, it is of the utmost importance to adjust glucocorticoid dose in situations with increased cortisol demand in order to prevent as well as appropriately treat adrenal crisis.

Conclusions: To prevent life-threatening adrenal crisis, patients, their families and medical staff require training.

\section{Keywords}

Hydrocortisone · Adrenal crisis · Emergency card · COVID-19 - Mineralocorticoids

risk profile as hydrocortisone in glucocorticoid replacement. Endocr Connect 6:766-772

31. https://www.glandula-online.de/brauche-icheinen-notfallausweis.Zugegriffen:27.Okt. 2021
32. Quinkler M, Nilsen RM,ZopfKetal (2015) Modifiedrelease hydrocortisone decreases $\mathrm{BMI}$ and $\mathrm{HbA1c}$ in patients with primary and secondary adrenal insufficiency. Eur J Endocrinol 172(5):619-626
Hier steht eine Anzeige. Springer 